\title{
PARAMETRIZATION OF GENERAL SOLUTIONS FOR THE NAVIER-STOKES EQUATIONS
}

\author{
BY \\ K. B. RANGER \\ University of Toronto, Toronto, Canada
}

\begin{abstract}
A method is described for constructing general solutions of the steady two-dimensional Navier-Stokes equations governing the motion of a viscous incompressible liquid. The solution for the stream function is expressed in implicit parametric form containing two arbitrary complex functions or four arbitrary real functions.
\end{abstract}

Introduction. To determine quantities of physical interesi for the steady motion of a viscous incompressible liquid such as force, torque, pressure, boundary vorticity, or shear stress requires a solution for the fluid velocity satisfying the Navier-Stokes equations. The flow equations though quasi-linear and autonomous contain sufficient nonlinearity, through coupling of the fluid velocity and vorticity, in such a way that solutions of generality can only be found implicitly defined through the use of parametric functions; see [2]. However, these implicit representations for the solutions, although not of the most desirable form, are not without interest and can be employed to investigate and simplify the boundary values of the basic flow quantities and their derivatives.

The present paper addresses the problem of constructing general type solutions that are implicitly defined in terms of parametric equations. The starting point is a concise complex variable formulation; see [1] for the simplest nondegenerate form of the Navier-Stokes equations; namely, the steady two-dimensional flow equations governing the motion of viscous incompressible liquid. This complex equation contains the stream function $\psi$ and an auxiliary real function $\phi$ as dependent variables and has the advantages of being quasi-linear, autonomous, with the only independent variable being $\bar{z}=x-i y$. All three properties are used in the present analysis. The method of solution is to parametrize $\psi$ and $\phi$ in terms of $\phi_{z}, \phi_{\bar{z}}, \psi_{z}, \psi_{\bar{z}}$, and this is achieved by the introduction of complex stream functions which provide nonlinear equations of Riccati type and allow explicit integration. The complex stream functions have no obvious physical significance and are used as an analytical device to determine the real physical stream function $\psi$. In general, neither the real nor the imaginary parts of the complex stream functions are solutions of the 
real flow equations. By applying consistency or integrability conditions to the integrated Riccati equations and the complex flow equation, a full parametric solution to the Navier-Stokes equations, containing two arbitrary complex functions, is obtained. The biharmonic solution for Stokes flow is determined in the limit kinematic viscosity $\nu \rightarrow \infty$.

The equations of motion. The equations describing the steady motion of a viscous incompressible liquid are given by

$$
\begin{gathered}
-[\mathbf{q} \times \operatorname{curl} \mathbf{q}]=-\operatorname{grad} B+\nu \nabla^{2} \mathbf{q}, \\
B=\frac{p}{\rho}+\frac{1}{2}\left[\left.\mathbf{q}\right|^{2}, \quad \operatorname{div} \mathbf{q}=0,\right.
\end{gathered}
$$

where $\mathbf{q}$ is the fluid velocity, $p$ the pressure, $\rho$ the density, $\nu$ the kinematic viscosity, and $B$ the Bernoulli function. Here part of the usual convection term $(\mathbf{q} \cdot \nabla \mathbf{q})$ has been absorbed into the Bernoulli function. For a two-dimensional flow there is a stream function defined by

$$
\mathbf{q}=u \hat{i}+v \hat{j}=\operatorname{curl}(-\psi \hat{k})=-\psi_{y} \hat{i}+\psi_{x} \hat{j}
$$

There is a concise complex variable formulation of Eqs. (1) and (2) first given in [1] and subsequently discovered by others and given by

$$
\begin{gathered}
\phi_{\overline{z z}}+i \psi_{\overline{z z}}+\frac{1}{2 \nu} \psi_{\bar{z}}^{2}=0, \quad z=x+i y, \quad \bar{z}=x-i y, \\
B=-\nu \nabla^{2} \phi=-4 \nu \phi_{z \bar{z}}=\frac{p}{\rho}+2 \psi_{z} \psi_{\bar{z}},
\end{gathered}
$$

where $\phi$ is a real function of $x, y$ playing an auxiliary role in defining the equations and has no real physical significance. The complex conjugate equation is

$$
\phi_{z z}-i \psi_{z z}+\frac{1}{2 \nu} \psi_{z}^{2}=0
$$

Elimination of $\phi$ by differentiation yields the usual vorticity equation

$$
\nu \nabla^{4} \psi=\frac{\partial\left(\psi, \nabla^{2} \psi\right)}{\partial(x, y)} .
$$

The only known general solution of Eq. (4) for the stream function $\psi$ is provided by

$$
\psi=k\left(x^{2}+y^{2}\right)+\phi_{1}(x, y), \quad \nabla^{2} \phi_{1}=0,
$$

and $k$ a real constant. The formal limit as $\nu \rightarrow \infty$ of the complex flow equations is

$$
\phi_{\overline{z z}}+i \psi_{\overline{z z}}=0
$$

for which the solution is

$$
\phi+i \psi=\bar{z} f(z)+g(z),
$$

where $f(z), g(z)$ are analytic in a suitable region of the fluid. This result will be recovered later as a by-product limit of nonlinear analysis. 
It is observed that the complex flow equation (4) can also be expressed as a complex Schrödinger equation in the form

$$
\Gamma_{\overline{z z}}=\frac{1}{2 \nu} \phi_{\overline{z z}} \cdot \Gamma, \quad \Gamma=e^{\psi /(2 \nu i)},
$$

but the nonlinearity is not circumvented since $\Gamma \bar{\Gamma}=1$.

Method of solution. It is appropriate to define

$$
\begin{aligned}
L & \equiv \phi_{\overline{z z}}+i \psi_{\overline{z z}}+\frac{1}{2 \nu} \psi_{\bar{z}}^{2}, \\
L_{1} & \equiv \phi_{\overline{z z}}+i F_{\overline{z z}}+\frac{1}{2 \nu} F_{\bar{z}}^{2}, \\
L_{2} & \equiv \phi_{\overline{z z}}+i G_{\overline{z z}}+\frac{1}{2 \nu} G_{\bar{z}}^{2},
\end{aligned}
$$

where $\phi \equiv \phi(x, y), \psi \equiv \psi(x, y)$ are real functions of $x, y$ and $F \equiv F(z, \bar{z})$, $G \equiv G(z, \bar{z})$ are complex functions of $z, \bar{z}$. It will appear that $F, G$ can be interpreted as complex stream functions with no immediate physical significance and are essentially used as analytic artifices in constructing the real physical stream function $\psi$.

The equation

$$
L_{1}-L \equiv i e^{(-F-\psi) /(2 \nu i)} \frac{\partial}{\partial \bar{z}}\left\{e^{(F+\psi) /(2 \nu i)}\left(F_{\bar{z}}-\psi_{\bar{z}}\right)\right\}=0
$$

is of Riccati type and implies

$$
e^{(F+\psi) /(2 \nu i)}\left(F_{\bar{z}}-\psi_{\bar{z}}\right)=f(z) .
$$

In a similar way the equation

$$
L_{2}-L \equiv i e^{(-G-\psi) /(2 \nu i)} \frac{\partial}{\partial \bar{z}}\left\{e^{(G+\psi) /(2 \nu i)}\left(G_{\bar{z}}-\psi_{\bar{z}}\right)\right\}=0
$$

implies

$$
e^{(G+\psi) /(2 \nu i)}\left(G_{\bar{z}}-\psi_{\bar{z}}\right)=g(z) .
$$

Also from Eqs. (15) and (17)

$$
f(z) e^{(G-\psi) /(2 \nu i)}\left(G_{\bar{z}}-\psi_{\bar{z}}\right)-g(z) e^{(F-\psi) /(2 \nu i)}\left(F_{\bar{z}}-\psi_{P \bar{z}}\right)=0,
$$

which permits an integration of the form

$$
f(z) e^{G /(2 \nu i)}-g(z) e^{F /(2 \nu i)}=h(z) e^{\psi /(2 \nu i)} .
$$

The functions $f(z), g(z), h(z)$ are analytic in a suitable region of the fluid. For consistency at a later stage it is expedient to choose

$$
h(z)=1, \quad g(z)=f(z)
$$

so that

$$
f(z)\left\{e^{G /(2 \nu i)}-e^{F /(2 \nu i)}\right\}=e^{\psi /(2 \nu i)} .
$$

Also Eq. (15) implies $L_{1}-L=0$ and Eq. (21) implies

$$
e^{G /(2 \nu i)}\left(L_{2}-L\right)-e^{F /(2 \nu i)}\left(L_{1}-L\right)=0 .
$$


Again from Eqs. (15) and (17)

$$
\begin{aligned}
G_{\bar{z}}-F_{\bar{z}} & =f(z)\left[e^{(-G-\psi) /(2 \nu i)}-e^{(-F-\psi) /(2 \nu i)}\right] \\
& =f(z) e^{(-G-F) /(2 \nu i)}\left[e^{(F-\psi) /(2 \nu i)}-e^{(G-\psi) /(2 \nu i)}\right] \\
& =-e^{(-G-F) /(2 \nu i)}
\end{aligned}
$$

so that

$$
e^{(G+F) /(2 \nu i)}\left(G_{\bar{z}}-F_{\bar{z}}\right)=-1,
$$

which implies

$$
L_{1}-L_{2}=0 \text {. }
$$

It will now be shown that there is a solution to the flow equations of the form

$$
G=F+K(\Omega), \quad \Omega=\phi+\alpha F,
$$

where $K$ is essentially an arbitrary complex function of its argument and $\alpha \neq i$ is a complex constant. Equation (24) simplifies to the equation

$$
e^{[2 F+K(\Omega)] /(2 \nu i)} K^{\prime}(\Omega) \Omega_{\bar{z}}+1=0 .
$$

For the following analysis it is convenient to regard $\phi, \psi$ as functions of $F, \bar{F}$ and write

$$
F_{\bar{z}}=M, \quad \bar{F}_{\bar{z}}=N,
$$

where $M \equiv M(F, \bar{F}), N \equiv N(F, \bar{F})$ are complex functions of $F, \bar{F}$. The integrability condition $F_{z \bar{z}}=F_{\bar{z} z}$ provides the complex partial differential equation

$$
M_{F} \bar{N}+M_{\bar{F}} \bar{M}=\bar{N}_{F} M+\bar{N}_{\bar{F}} N,
$$

and Eqs. (27) reduces to

$$
e^{[2 F+K(\Omega)] /(2 \nu i)} K^{\prime}(\Omega)\left\{\left(\phi_{F}+\alpha\right) M+\phi_{\bar{F}} N\right\}+1=0 .
$$

Now if $A, B, C$ are complex functions of $F, \bar{F}$, the equation

$$
A L_{1}+B L_{2}+C L=0
$$

is equivalent to

$$
\begin{gathered}
A\left(\phi_{\bar{z}}+i F_{\bar{z}}\right)+B\left(\phi_{\bar{z}}+i G_{\bar{z}}\right)+C\left(\phi_{\bar{z}}+i \psi-\bar{z}\right)=1, \\
\frac{A}{2 \nu} F_{\bar{z}}^{2}+\frac{B}{2 \nu} G_{\bar{z}}^{2}+\frac{C}{2 \nu} \psi_{\bar{z}}^{2}-A_{\bar{z}}\left(\phi_{\bar{z}}+i F_{\bar{z}}\right)-B_{\bar{z}}\left(\phi_{\bar{z}}+i G_{\bar{z}}\right)-C_{\bar{z}}\left(\phi_{\bar{z}}+i \psi_{\bar{z}}\right)=0 .
\end{gathered}
$$

With $G=F+K(\Omega)$ and $F_{\bar{z}}=M, \bar{F}_{\bar{z}}=N$, these equations can be written as

$$
\begin{aligned}
\left.A\left[\left(\phi_{F}+i\right) M+\phi_{\bar{F}} N\right]+B\left\{\left(\phi_{F}+i\right) M+\phi_{\bar{F}} N+K^{\prime}(\Omega)\left[\left(\phi_{F}+\alpha\right) M+\phi_{\bar{F}} N\right)\right]\right\} & +C\left[\left(\phi_{F}+i \psi_{F}\right) M+\left(\phi_{\bar{F}}+i \psi_{\bar{F}}\right) N\right]=1 \\
& \frac{A}{2 \nu} M^{2}+\frac{B}{2 \nu}\left\{M+K^{\prime}(\Omega)\left[\left(\phi_{F}+\alpha\right) M+\phi_{\bar{F}} N\right]\right\}^{2}+\frac{C}{2 \nu}\left[\psi_{F} M+\psi_{\bar{F}} N\right]^{2} \\
& -\left(A_{F} M+A_{\bar{F}} N\right)\left[\left(\phi_{F}+i\right) M+\phi_{\bar{F}} N\right] \\
& -\left(B_{F} M+B_{\bar{F}} N\right)\left\{\left(\phi_{F}+i\right) M+\phi_{\bar{F}} N+K^{\prime}(\Omega)\left[\left(\phi_{F}+\alpha\right) M+\phi_{\bar{F}} N\right]\right\} \\
& -\left(C_{F} M+C_{\bar{F}} N\right)\left\{\left(\phi_{F}+i \psi_{F}\right) M+\left(\phi_{\bar{F}}+i \psi_{\bar{F}}\right) N\right\}=0 .
\end{aligned}
$$


From Eqs. (30) and (34), $M, N$ can be expressed in terms of $A, B, C$, and eliminating $M, N$ from Eqs. (29) and (35) yields two complex partial differential equations for the complex functions $A, B, C$. In view of the arbitrary nature of $K(\Omega)$ and that there are three complex dependent variables defined by two complex partial differential equations (29), (35), it is in general possible to satisfy these equations for $A, B, C$ in such a way that $A+B+C \neq 0$ and $\phi \equiv \phi(F, F), \psi \equiv \psi(F, \bar{F})$ are essentially unrestricted. In this case the equations

$$
\begin{gathered}
L_{1}-L_{2}=0, \quad e^{G /(2 \nu i)}\left(L_{2}-L\right)-e^{F /(2 \nu i)}\left(L_{1}-L\right) \equiv 0, \\
A L_{1}+B L_{2}+C L=0, \quad A+B+C \neq 0, \quad G=F+K(\Omega),
\end{gathered}
$$

imply $L=L_{1}=L_{2}=0$ and the flow equations have a solution with $G=F+K(\Omega)$, $\Omega=\phi+\alpha F$.

The success of this method depends on the basic quasi-linearity of the flow equations. It then follows from Eqs. (36) and (37) that

$$
\begin{gathered}
f(z)\left[e^{[F+K(\Omega)] /(2 \nu i)}-e^{F /(2 \nu i)}\right]-e^{\psi /(2 \psi i)}=0, \\
e^{[2 F+K(\Omega)] /(2 \nu i)} K^{\prime}(\Omega) \Omega_{\bar{z}}+1=0
\end{gathered}
$$

have solutions consistent with $L=0$ in which $f(z)$ and $K(\Omega)$ are unrestricted. Since from Eq. (38)

$$
e^{(G-F) /(2 \nu i)} f(z)-f(z)=e^{(\psi-F) /(2 \nu i)}, \quad G=F+K(\Omega),
$$

it follows that

$$
e^{(G-F) /(2 \nu i)}\left(G_{\bar{z}}-F_{\bar{z}}\right) f(z)=e^{(\psi-F) /(2 \nu i)}\left(\psi_{\bar{z}}-F_{\bar{z}}\right),
$$

which in turn implies from Eq. (24) that

$$
e^{(F+\psi) /(2 \nu i)}\left(F_{\bar{z}}-\psi_{\bar{z}}\right)=f(z) .
$$

Again Eq. (38) is equivalent to

$$
F-\psi+2 \nu i \log f(z)=S(\omega), \quad \omega=\phi+\alpha \psi-2 \nu \alpha i \log f(z),
$$

where $S$ is an arbitrary function of its argument since $K$ is arbitrary. Eliminating $F$ from Eqs. (42) and (43) yields

$$
e^{(2 \psi+S(\omega)) /(2 \nu i)} S^{\prime}(\omega) \omega_{\bar{z}}=(f(z))^{2}, \quad \omega=\phi+\alpha \psi-2 \nu \alpha i \log f(z),
$$

which can be written in the form

$$
\omega_{\bar{z}}=j(z) M_{1}(\omega) e^{-\psi /(\nu i)}, \quad M_{1}(\omega)=\frac{e^{-S(\omega) /(2 \nu i)}}{S^{\prime}(\omega)}, \quad j(z)=(f(z))^{2},
$$

and $\omega=\phi+\alpha \psi-\nu \alpha i \log j(z)$. It remains to apply integrability conditions in conjunction with the complex flow equation $L=0$. This can be simplified by setting

$$
\psi_{\bar{z}}=f(z) N_{1}(\omega) e^{\psi /(\nu i)},
$$


and from Eq. (45) the equation $L=0$ is satisfied providing

$$
\begin{aligned}
& {\left[M_{1}^{\prime}(\omega)+(i-\alpha) N_{1}^{\prime}(\omega)\right] M_{1}(\omega)-\frac{1}{\nu i} N_{1}(\omega)\left[M_{1}^{\prime}(\omega)+(i-\alpha) N_{1}^{\prime}(\omega)\right]} \\
& \quad+\frac{1}{2 \nu}\left[N_{1}(\omega)\right]^{2}=0 .
\end{aligned}
$$

By writing $M_{1}(\omega)=R(\omega) N_{1}(\omega), N_{1}(\omega)$ can be expressed in terms of $R(\omega)$ by the formula

$$
N_{1}(\omega)=\frac{A_{0}}{[R(\omega)+i-\alpha]} e^{\frac{\omega}{\nu i}-\frac{1}{2 \nu} \int d \omega /[R(\omega)+i-\alpha]},
$$

where $A_{0}$ is a complex constant. Both $M_{1}(\omega)$ and $N_{1}(\omega)$ can now be expressed in terms of the arbitrary complex function $R(\omega)$. The integrability conditions $\phi_{z \bar{z}}=$ $\phi_{\bar{z} z}, \psi_{z \bar{z}}=\psi_{\bar{z} z}$ with $\phi, \psi$ real lead to the real equations

$$
\begin{aligned}
& j^{\prime}(z)\left[M_{1}(\omega)-\alpha N_{1}(\omega)\right] e^{-\psi /(\nu i)}+j(z) e^{-\psi /(\nu i)}\left[M_{1}^{\prime}(\omega)-\alpha N_{1}^{\prime}(\omega)\right] \\
& \times\left\{\bar{j}(\bar{z}) e^{\psi /(\nu i)}\left[\bar{M}_{1}(\bar{\omega})-\bar{\alpha} \bar{N}_{1}(\bar{\omega})\right]+\alpha \bar{j}(\bar{z}) e^{\psi /(\nu i)} \bar{N}_{1}(\bar{\omega})-\nu \alpha i \frac{j^{\prime}(z)}{j(z)}\right\} \\
& -\frac{j(z)}{\nu i}\left[M_{1}(\omega)-\alpha N_{1}(\omega)\right] \bar{j}(\bar{z}) \bar{N}_{1}(\bar{\omega}) \\
& =\bar{j}^{\prime}(\bar{z}) e^{\psi /(\nu i)}\left[\bar{M}_{1}(\bar{\omega})-\bar{\alpha} \bar{N}_{1}(\bar{\omega})\right]+\bar{j}(\bar{z}) e^{\psi /(\nu i)}\left[\bar{M}_{1}^{\prime}(\bar{\omega})-\bar{\alpha} \bar{N}_{1}^{\prime}(\bar{\omega})\right] \\
& \quad \times\left\{j(z) e^{-\psi /(\nu i)}\left[M_{1}(\omega)-\alpha N_{1}(\omega)\right]+\bar{\alpha} j(z) e^{-\psi /(\nu i)} N_{1}(\omega)+\nu \bar{\alpha} i \frac{\bar{j}^{\prime}(\bar{z})}{\bar{j}(\bar{z})}\right\} \\
& \quad+\frac{\bar{j}(\bar{z})}{\nu i}\left[\bar{M}_{1}(\bar{\omega})-\bar{\alpha} \bar{N}_{1}(\bar{\omega})\right] j(z) N_{1}(\omega)
\end{aligned}
$$

and

$$
\begin{aligned}
& j^{\prime}(z) N_{1}(\omega) e^{-\psi /(\nu i)}+j(z) e^{-\psi /(\nu i)} N_{1}^{\prime}(\omega) \\
& \times\left\{\bar{j}(\bar{z}) e^{\psi /(\nu i)}\left[\bar{M}_{1}(\bar{\omega})-\bar{\alpha}_{1}(\bar{\omega})\right]+\alpha \bar{j}(\bar{z}) e^{\psi /(\nu i)} \bar{N}_{1}(\bar{\omega})-\alpha \nu i \frac{j^{\prime}(z)}{j(z)}\right\} \\
& -\frac{j(z)}{\nu i} N_{1}(\omega) \bar{j}(\bar{z}) \bar{N}_{1}(\bar{\omega}) \\
& =\bar{j}^{\prime}(\bar{z}) \bar{N}_{1}(\bar{\omega}) e^{\psi /(\nu i)}+\bar{j}(\bar{z}) e^{\psi /(\nu i)} \bar{N}_{1}^{\prime}(\bar{\omega}) \\
& \quad \times\left\{j(z) e^{-\psi /(\nu i)}\left[M_{1}(\omega)-\alpha N_{1}(\omega)\right]+\bar{\alpha} j(z) e^{-\psi /(\nu i)} N_{1}(\omega)+\bar{\alpha} \nu i \frac{\bar{j}^{\prime}(\bar{z})}{\bar{j}(\bar{z})}\right\} \\
& \quad+\frac{\bar{j}(\bar{z})}{\nu i} \bar{N}_{1}(\bar{\omega}) j(z) N_{1}(\omega) .
\end{aligned}
$$

Equations (49) and (50) represent a general solution of the steady flow equations in which $\phi, \psi$ are implicitly defined with $j(z)$ and $R(\omega)$ essentially arbitrary. This completes the analysis except to check the results in the limiting case $\nu \rightarrow \infty$, which results in Stokes flow, and also to recover potential flow as an exact solution. 
Stokes or creeping flow. As a check on the preceding analysis, it is useful to return to Eq. (44) in the form

$$
e^{[2 \psi+S(\omega)] /(2 \nu i)} S^{\prime}(\omega) \omega_{\bar{z}}=j(z), \quad \omega=\phi+\alpha \psi-\nu \alpha i \log j(z) ;
$$

taking the formal limit as $\nu \rightarrow \infty$ of $L=0$ and Eq. (51) results in the consistent solution

$$
\phi_{\bar{z}}+i \psi_{\bar{z}}=m(z), \quad m(z)=j(z) e^{\beta_{0} \log j(z)},
$$

where

$$
\lim _{\nu \rightarrow \infty} \alpha=i, \quad \beta_{0}=\lim _{\nu \rightarrow \infty}\left(\frac{\alpha \beta i}{2}\right),
$$

and $S(\omega)=\beta \omega, \beta$ a complex constant. An arbitrary function $S(\omega)$ is lost in the limiting process but is recovered by integration to provide

$$
\phi+i \psi=\bar{z} m(z)+n(z)
$$

or equivalently

$$
\begin{aligned}
2 \phi & =\bar{z} m(z)+4 z \bar{m}(\bar{z})+n(z)+\bar{n}(z), \\
2 i \psi & =\bar{z} m(z)-z \bar{m}(\bar{z})+n(z)-\bar{n}(\bar{z}),
\end{aligned}
$$

which are clearly both biharmonic functions.

Potential flow. As a second check on the analysis it is noted that a general potential flow is obtained from Eq. (49) with $S(\omega)=$ constant and $j(z)=0$. The equation $L=0$ is then satisfied by

$$
\phi=a(z)+\bar{a}(\bar{z}), \quad \psi=b(z)+\bar{b}(\bar{z}),
$$

with

$$
\bar{a}^{\prime \prime}(\bar{z})+i \bar{b}^{\prime \prime}(\bar{z})+\frac{1}{2 \nu}\left(\bar{b}^{\prime}(\bar{z})\right)^{2}=0
$$

\section{REFERENCES}

[1] R. Legendre, Solutions plus complète du problème Blasius, Comptes Rendus, Tom. 228, June 1949, pp. 2008-2010

[2] K. B. Ranger, A complex variable integration technique for the two-dimensional Navier-Stokes equations, Quart. Appl. Math. XLIX, 555-562 (1991) 